\title{
NUMERICAL SIMULATION OF NONPENETRATIVE TURBULENT THERMAL CONVECTION
}

\author{
Ajay K. Prasad \\ Department of Mechanical Engineering, University of Delaware, Newark, \\ Delaware 19716-3140, USA
}

\begin{abstract}
Nonpenetrative convection-in which the lower boundary condition is one of constant heat flux and the upper boundary is insulated-is an idealization of penetrative convection, which finds application in the planetary boundary layer. Three-dimensional, unsteady, direct numerical simulations $(96 \times 96 \times 128)$ have been performed of turbulent, nonpenetrative thermal convection inside a cubical enclosure at $R a=2 \times 10^{7}$. While the bulk temperature of the fluid increases linearly with time, the spatially averaged temperature profile exhibits a time-invariant behavior along the vertical coordinate. The numerical study has replicated results from earlier Particle Image Velocimetry (PIV) measurements of the same flow, in a $76.2 \mathrm{~mm}$ thick layer of water, at an aspect ratio of 6.6. Time-averaged vertical profiles of mean, root-mean-square, and skewness of velocity and temperature profiles compare well with experiments. Instantaneous velocity maps and temperature contours are also in good agreement with previous experimental results. Finally, while both the upper and lower boundary temperatures increase linearly with time, their difference is constant and matches well with analytical predictions.
\end{abstract}

\section{INTRODUCTION}

A standard laboratory model used by meteorologists consists of a shallow layer of water, heated from below and capped from above by a stably stratified layer $[1,2]$. The stable layer represents the inversion that caps the turbulent motions in the atmosphere. Over the course of the experiment, the heating at the lower boundary drives the turbulent motions in the form of plumes and thermals, which rise through the depth of the mixed or convecting layer and impact the base of the stable layer, and gradually erode it away. This laboratory model mimics the gradual erosion of the inversion layer in the atmosphere over the course of the day, due to the action of thermally induced motions arising from solar heating at the earth's surface. The form of convection described above is called penetrative convection, alluding to the penetration of the turbulent structures from the mixed layer into the stable inversion layer. It should be noted that a desk-top experiment using water as the working fluid obviously precludes a direct comparison to the atmosphere owing to the huge disparity in scales and the inability to incorporate complicating factors of compressibility and moist convection; yet, the chosen configuration has repeatedly proved its utility by providing physical in-

Received 10 August 1998; revised 24 October 1998.

This work was initiated during a visit to the Laboratory for Aero and Hydrodynamics, Delft University of Technology, during summer 1997. The basic simulation code was provided by Professor Frans Nieuwstadt's group. The author gratefully acknowledges the help provided by Dr. B. J. Boersma, Mr. B. van Haarlem, and Dr. Jerry Westerweel.

Address correspondence to Professor Ajay K. Prasad, Department of Mechanical Engineering, University of Delaware, 126 Spencer Laboratory, Newark, DE 19716-3140, USA. 


\section{NOMENCLATURE}

$C_{P} \quad$ specific heat

$g \quad$ acceleration due to gravity

$x, y, z \quad$ spatial coordinates

acceleration due to gravity
Grashof number

$z_{*} \quad$ depth of fluid layer

$\begin{array}{ll}\mathrm{Gr} & \text { Grasified Grashof number } \\ \mathrm{Gr}^{\prime} & \text { modified }\end{array}$

$k$

thermal conductivity

$\alpha \quad$ thermal diffusivity

$\beta \quad$ thermal expansion coefficient

$\theta \quad$ nondimensional temperature

$\mathrm{Nu} \quad$ Nusselt number

$p \quad$ pressure

Pr Prandtl number

$q \quad$ heat flux, $\mathrm{W} / \mathrm{m}^{2}$

$q_{0}$ heat flux at lower boundary, $\mathrm{W} / \mathrm{m}^{2}$

$\mathrm{Ra}$ Rayleigh number

$\mathrm{Ra}^{\prime} \quad$ modified Rayleigh number

$\mathrm{Re} \quad$ Reynolds number

$t$ time

$T \quad$ temperature

$u, v, w \quad$ velocity components long $(x, y, z)$

$w_{*}$ convective velocity

$\lambda_{T} \quad$ thickness of conduction layer

$v \quad$ kinematic viscosity

$\rho$ density

$\sigma \quad$ rms quantity

\section{Subscripts}

$b \quad$ lower boundary (bottom) of fluid layer

$m \quad$ mixed layer

* convective scale

sight into atmospheric phenomena and certain results that match atmospheric measurements.

An idealization of such a penetrative configuration is obtained by replacing the stable inversion layer with a rigid, insulating boundary, a situation called nonpenetrative convection (Figure 1). In this idealized case, turbulent motions generated at the lower boundary propagate through the mixed layer as before but cannot, of course, penetrate the rigid cap, hence the nonpenetrative label. Nonpenetrative convection also occurs in lakes or oceans, where the surface layer is evaporatively cooled, and the bottom of the fluid layer forms the rigid, insulating boundary. In the latter case, the convection motions (plumes) are oriented from top-to-bottom, in contrast to the bottom-to-top motions in the atmosphere; Wyngaard and Weil [3] refer to these two situations as top-down and bottom-up, respectively.

The subject of this paper pertains to bottom-up nonpenetrative convection in which the turbulent motions are driven by heating from the bottom. A constant heat flux is applied at the lower boundary, and the upper boundary is insulated. The simulated conditions replicate earlier experiments by Prasad and Gonuguntla [4], where a $76.2 \mathrm{~mm}$ layer of water in a square enclosure of side $50 \mathrm{~cm}$ (aspect

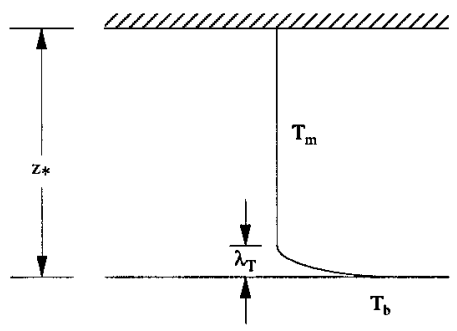

Figure 1. Temperature profile in nonpenetrative thermal convection; all temperatures increase linearly in time; however, the spatial distribution is steady. 
ratio 6.6) was driven from below with an electrical heater at a rate giving a Rayleigh number $\mathrm{Ra}$ of $2 \times 10^{7}$. The only difference is that in the current simulation the aspect ratio is fixed at unity. Direct numerical simulations were performed using the three-dimensional, time-dependent momentum and energy equations. The results are in good agreement with the experimental measurements. The simulations also provide verification of an analytical prediction obtained by Prasad [5] for the (constant) temperature difference between the upper and lower boundaries of the enclosure. The literature lists only a few other studies of nonpenetrative convection: Adrian et al. [6] performed point-wise temperature and velocity measurements in a shallow layer of water; Moeng and Rotunno [7] performed $96^{3}$ direct numerical simulations albeit at a substantially smaller Ra (they used $\mathrm{Nu}=7$ and $\mathrm{Pra}=1$ in their work).

\section{GOVERNING EQUATIONS}

Momentum and energy equations were solved simultaneously in a time dependent manner using a finite difference formulation. (Professor F. Nieuwstadt at TU Delft, Netherlands, provided the original code, which was suitably modified for this work.) The fluid properties were assumed to be constant; the Boussinesq approximation was applied to restrict the variation of density with temperature exclusively in the body force term. The corresponding equations are as follows.

\section{Momentum}

$$
\frac{\partial u_{i}}{\partial t}+u_{j} \frac{\partial u_{i}}{\partial x_{j}}=-\frac{1}{\rho_{s}} \frac{\partial p}{\partial x_{i}}+v \frac{\partial^{2} u_{i}}{\partial x_{j}^{2}}+\left(T-T_{s}\right) \beta g \delta_{i 3}
$$

In the above equation, $T_{s}$ is the reference temperature that was chosen as the starting or initial temperature, $\rho_{s}$ is the fluid density at $T_{s}, \beta$ is the thermal expansion coefficient, $v$ is the kinematic viscosity, $u_{i}$ is the velocity component along the $x_{i}$ coordinate, $p$ is the pressure, $T$ is the temperature, and $g$ is the gravitational constant. In subsequent sections, we will use $u$ and $v$ to represent the horizontal velocity components, while $w$ denotes the vertical velocity component; similarly, $x$ and $y$ denote the horizontal coordinates and $z$ denotes the vertical coordinate.

Energy

$$
\frac{\partial T}{\partial t}+u_{j} \frac{\partial T}{\partial x_{j}}=\alpha \frac{\partial^{2} T}{\partial x_{j}^{2}}
$$

where $\alpha$ is the thermal diffusivity of the fluid.

\section{Nondimensional Scheme}

All distances are normalized by the depth of the fluid layer $z_{*}$. All velocities are normalized by the convective velocity scale $w_{*}$, which is defined as

$$
w_{*}=\left[\frac{g \beta q_{0} z_{*}}{\rho_{s} C_{p}}\right]^{1 / 3}
$$


where $q_{0}$ is the constant heat flux $\left(\mathrm{W} / \mathrm{m}^{2}\right)$ supplied to the lower boundary and $C_{p}$ is the specific heat.

The nondimensional temperature is written as

$$
\theta=\left(T-T_{s}\right) \frac{k}{q_{0} z_{*}}
$$

where $k$ is the thermal conductivity of the fluid. At the start of the simulation, $\theta=0$ because $T=T_{s}$.

Time is nondimensionalized as $t^{\prime}$, the convective timescale:

$$
t^{\prime}=t \frac{w_{*}}{z_{*}}
$$

such that typical convective motions traverse the depth of the fluid in one nondimensional timescale.

Pressure is nondimensionalized as $p^{\prime}$ :

$$
p^{\prime}=\frac{p}{\rho_{0} w_{*}^{2}}
$$

\section{Nondimensional Governing Equations}

Using the nondimensional scheme, the governing equations may be written as follows. Note that all primes have been dropped for convenience.

Nondimensional momentum

$$
\frac{\partial u_{i}}{\partial t}+u_{j} \frac{\partial u_{i}}{\partial x_{j}}=-\frac{\partial p}{\partial x_{i}}+\frac{1}{\operatorname{Re}} \frac{\partial^{2} u_{i}}{\partial x_{j}^{2}}+\frac{\mathrm{Gr}^{\prime}}{\mathrm{Re}^{2}} \theta \delta_{i 3}
$$

\section{Nondimensional energy}

$$
\frac{\partial \theta}{\partial t}+u_{j} \frac{\partial \theta}{\partial x_{j}}=\frac{1}{\operatorname{Re} \operatorname{Pr}} \frac{\partial^{2} \theta}{\partial x_{j}^{2}}
$$

where $\mathrm{Re}$ is the Reynolds number, $\mathrm{Pr}$ is the Prandtl number, and $\mathrm{Gr}^{\prime}$ is a modified Grashof number. We use $\mathrm{Gr}^{\prime}$ as opposed to $\mathrm{Gr}$ because our definition of the Grashof number (see below) involves the heat flux $q_{0}$ in contrast to the conventional definition involving a temperature differential. The reason for this alternative definition is that in our configuration the heat flux $q_{0}$ is the prescribed quantity:

$$
\begin{aligned}
\mathrm{Re} & =\frac{w_{*} z_{*}}{v} \\
\mathrm{Gr}^{\prime} & =\frac{g \beta z_{*}^{3}}{v^{2}} \frac{q_{0} z_{*}}{k}
\end{aligned}
$$


Using $\mathrm{Ra}^{\prime}=\mathrm{Gr}^{\prime} \mathrm{Pr}$, where $\mathrm{Ra}^{\prime}$ is a modified Rayleigh number, it is easily shown that

$$
\begin{aligned}
\operatorname{Re} & =\left(\frac{\mathrm{Ra}^{\prime}}{\mathrm{Pr}^{2}}\right)^{1 / 3} \\
\operatorname{Re} \operatorname{Pr} & =\left(\mathrm{Ra}^{\prime} \operatorname{Pr}\right)^{1 / 3} \\
\frac{\mathrm{Gr}^{\prime}}{\mathrm{Re}^{2}} & =\left(\mathrm{Ra}^{\prime} \operatorname{Pr}\right)^{1 / 3}
\end{aligned}
$$

Thus all three nondimensional groupings in the momentum and energy equations (Re, $\mathrm{Re} \mathrm{Pr}$, and $\mathrm{Gr}^{\prime} / \mathrm{Re}^{2}$ ) may be specified completely by specifying only $\mathrm{Ra}^{\prime}$ and Pr. As stated in Ref. [5], $\mathrm{Ra}^{\prime}$ can significantly exceed $\mathrm{Ra}$ by 1 or 2 orders of magnitude even in laboratory-scale experiments, and therefore $\mathrm{Ra}^{\prime}$ cannot accurately convey the dynamic state of thermal convection. However, it is seen below that $\mathrm{Ra}$ is related very simply to $\mathrm{Ra}^{\prime}$ :

$$
\begin{aligned}
\mathrm{Ra}^{\prime} & =\frac{g \beta z_{*}^{3}}{v \alpha} \frac{q_{0} z_{*}}{k} \\
& =\left(\frac{g \beta \Delta T z_{*}^{3}}{v \alpha}\right)\left(\frac{q_{0} z_{*}}{k \Delta T}\right) \\
& =\mathrm{RaNu}
\end{aligned}
$$

In other words, our modified Rayleigh number $\mathrm{Ra}^{\prime}$, is equal to the conventional Rayleigh number $\mathrm{Ra}$ multiplied by the Nusselt number $\mathrm{Nu}$; $\mathrm{Nu}$ can reach values in the hundreds even in the laboratory experiments mentioned in the Introduction. However, $\mathrm{Nu}$ is not known a priori. Consequently, while it is easy to prescribe $\mathrm{Ra}^{\prime}$ in nonpenetrative convection by prescribing the heat flux, the fluid, and the overall geometry of the enclosure, $\mathrm{Ra}$ can only be estimated after determining $\mathrm{Nu}$ from the simulations.

Prasad [5] provides a derivation for $\mathrm{Ra}$ in the nonpenetrative scenario using established correlations for Rayleigh-Benard convection and making the assumption that at high $\mathrm{Ra}$, the turbulence follows the classical scaling argument that $\mathrm{Nu} \sim \mathrm{Ra}^{1 / 3}$. The results from the current simulation provide a convenient database against which Prasad's $\llbracket 5 \rrbracket$ analytical predictions may be tested.

\section{Boundary and Initial Conditions}

The simulation enforced no-slip on all six walls. The temperature boundary conditions were zero heat flux at the upper boundary and the four side walls; for the lower boundary the nondimensional temperature gradient was specified:

$$
\left.\frac{\partial \theta}{\partial z}\right|_{z=0}=-1.0
$$


The initial temperature was fixed at zero over the entire three-dimensional domain, whereas the initial velocities were assigned small random values. These random values settled down rapidly by diffusion within a few time steps and evolved into temperature-driven motions easily recognized as plumes or thermals.

\section{SIMULATION CONDITIONS}

We simulated nonpenetrative convection corresponding to a cubical enclosure of dimension $z_{*}=76.2 \mathrm{~mm}$ with water as the working fluid; all fluid properties were evaluated at a reference temperature of $24^{\circ} \mathrm{C}$. The heat flux $q_{0}$ was set at $985 \mathrm{~W} / \mathrm{m}^{2}$. For these conditions, the convective velocity scale $w_{*}=3.52$ $\mathrm{mm} / \mathrm{s}$; the dimensional time may be obtained as $t=21.65 t^{\prime} \mathrm{s}$. This combination gave $\mathrm{Ra}^{\prime}=10^{9}$ and $\operatorname{Pr}=6.3$. Using the correlation provided by Prasad [5], $\mathrm{Nu} \approx 50$, and therefore $\mathrm{Ra}=\mathrm{Ra}^{\prime} / \mathrm{Nu} \approx 2 \times 10^{7}$.

The resolution of the simulation (in the vertical direction) was decided by estimating the thickness of the conduction layer $\left(\lambda_{T}\right)$ adjacent to the lower boundary. The Nusselt number is defined as

$$
\mathrm{Nu}=\frac{q_{0} z_{*}}{k\left(T_{b}-T_{m}\right)}
$$

where

$$
q_{0}=-\left.k \frac{\partial T}{\partial z}\right|_{z=0}
$$

Using Figure 1 and the estimate that $\mathrm{Nu} \approx 50$ for the simulated case,

$$
\frac{\lambda_{T}}{z_{*}}=\frac{1}{\mathrm{Nu}}=0.02
$$

Requiring three to four grid points within the conduction layer implied a vertical resolution of 128 . Thermal gradients in the horizontal directions are less severe; therefore 96 grid points were used in each of the horizontal directions. The grids were spaced uniformly in each of the three directions. The normal component of velocity was fixed at zero at each wall; the other two components of velocity, pressure, and temperature were computed on a grid that was staggered by half a grid spacing from the grid on which the normal velocity was computed. Our selection of vertical grid spacing compares favorably with the work of Kerr [8] and Balachandar et al. [9], both of whom used 96 grids in the vertical direction for numerical simulation of Rayleigh-Benard convection at about the same Ra.

Approximately 12,000 CRAY J90 seconds were required for each convective timescale (corresponding to $21.65 \mathrm{~s}$ of physical time). Simulations were carried out for a total of 28 nondimensional timescales, corresponding to about $10 \mathrm{~min}$ of physical time. Instantaneous velocity and temperature data were stored at intervals corresponding to about one convective timescale for subsequent processing. 


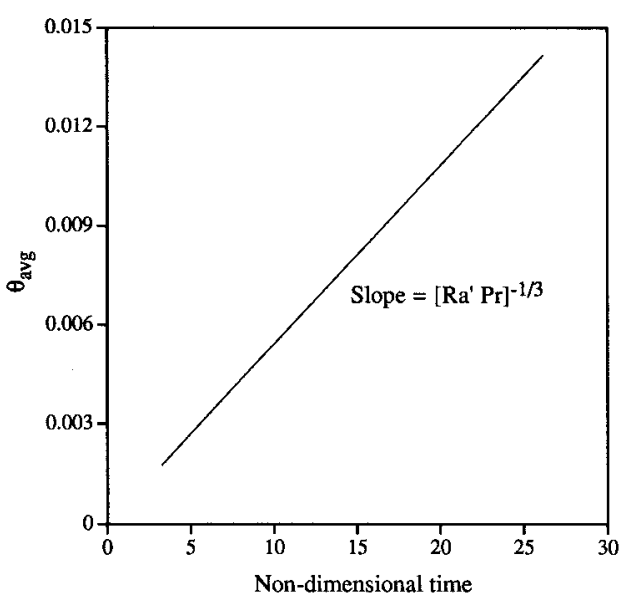

Figure 2. Variation of the average nondimensional temperature inside the enclosure $\left(\theta_{\text {avg }}\right)$ with time, confirming that the slope $=\left[\mathrm{Ra}^{\prime} \mathrm{Pr}\right]^{-1 / 3}$.

\section{RESULTS}

\section{General}

Figure 2 displays the time rate of change of the average or bulk nondimensional temperature inside the enclosure. As expected, the slope is linear because the rate of heat addition is constant. Energy balance requires that the heat addition to the fluid must raise its temperature linearly according to

$$
\frac{\partial T}{\partial t}=\frac{q_{0}}{\rho C_{p} z_{*}}
$$

Using the nondimensionalization described earlier, the above expression yields a nondimensional bulk temperature rise rate of

$$
\frac{\partial \theta}{\partial t}=\left[\operatorname{Ra}^{\prime} \operatorname{Pr}\right]^{-1 / 3}
$$

For the particular values of $\mathrm{Ra}^{\prime}$ and $\mathrm{Pr}$ chosen for this study, the nondimensional temperature rise rate should equal $5.414 \times 10^{-4}$. A least squares fit was performed to the data in Figure 2, which yielded a slope of $5.412 \times 10^{-4}$. This indicates the high degree to which energy is being conserved in our simulations.

Figure 3 displays four temperature time traces: (1) the average temperature over the lower boundary $\theta_{b}$, (2) the average or bulk temperature within the entire enclosure $\theta_{\text {avg }}$ (same as Figure 2), (3) the average temperature in the mixed layer $\theta_{m}$, and (4) the difference between the bottom and mixed layer temperatures $\left(\theta_{b}-\theta_{m}\right)$. The mixed layer was loosely defined as the region lying in the range $0.2<z / z_{*}<1.0$. Obviously, $\theta_{\text {avg }}$ slightly exceeds $\theta_{m}$ because the conduction layer, which the former includes, is much warmer than the mixed layer. 


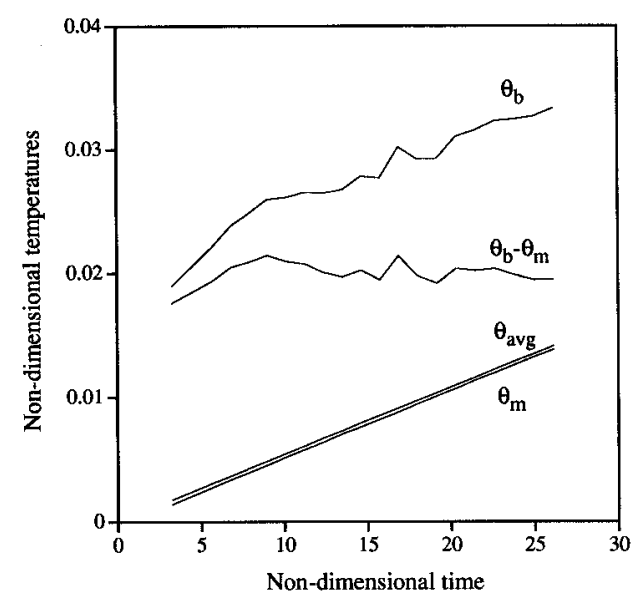

Figure 3. Variation of nondimensional temperatures with time: lower boundary $\theta_{b}$, mixed layer $\theta_{m}$, average across entire fluid layer $\theta_{\text {avg }}$, and temperature differential $\theta_{b}-\theta_{m}$.

Both $\theta_{\text {avg }}$ and $\theta_{m}$ increase linearly, as dictated by the energy balance. However, $\theta_{b}$ increases more rapidly during the initial stages (for $t^{\prime}<8$ ), after which it increases more or less linearly with a somewhat smaller slope. Note that all temperatures are zero at the start of the simulation. The initial higher slope is due to transients that exist in the period before which the turbulence may be considered "fully developed." Most important, we observe that the temperature difference $\left(\theta_{b}-\theta_{m}\right)$ attains a more or less constant value after the initial period. The average value of this differential from our simulations is 0.02 .

Prasad [5] derived an expression for the temperature differential $\left(T_{b}-T_{m}\right)$ is nonpenetrative convection according to

$$
q_{0}=0.174 \operatorname{Pr}^{0.074} k\left(\frac{g \beta}{v \alpha}\right)^{1 / 3}\left(T_{b}-T_{m}\right)^{4 / 3}
$$

By substituting for $q_{0}$, and the fluid properties in Eq. (10), we obtain $\left(T_{b}-T_{m}\right)=$ $2.33^{\circ} \mathrm{C}$. Converting to nondimensional form using Eq. (4), we finally obtain $\left(\theta_{b}-\theta_{m}\right)=0.019$, which is within $5 \%$ of the simulated value of 0.02 .

Horizontally averaged nondimensional temperature profiles are plotted in Figure 4 at selected times. A thin conduction layer is seen near the lower boundary, which contains all of the temperature variation. Outside the boundary layer the mixed region indicates a fairly uniform temperature owing to the rapid diffusion from turbulent motions.

As expected, the temperature profiles are very similar to each other and merely translate to the right as time increases. One may conclude therefore that the turbulence has developed into a quasi steady state in which (average) temperature unsteadiness is confined to a linear increase in time given by $\left(\mathrm{Ra}^{\prime} \mathrm{Pr}\right)^{-1 / 3}$, whereas the spatial profile is essentially time invariant. In fact, it is possible to collapse all temperature profiles onto a single curve by subtracting the time rate of change of the nondimensional temperature. The average of all such collapsed 

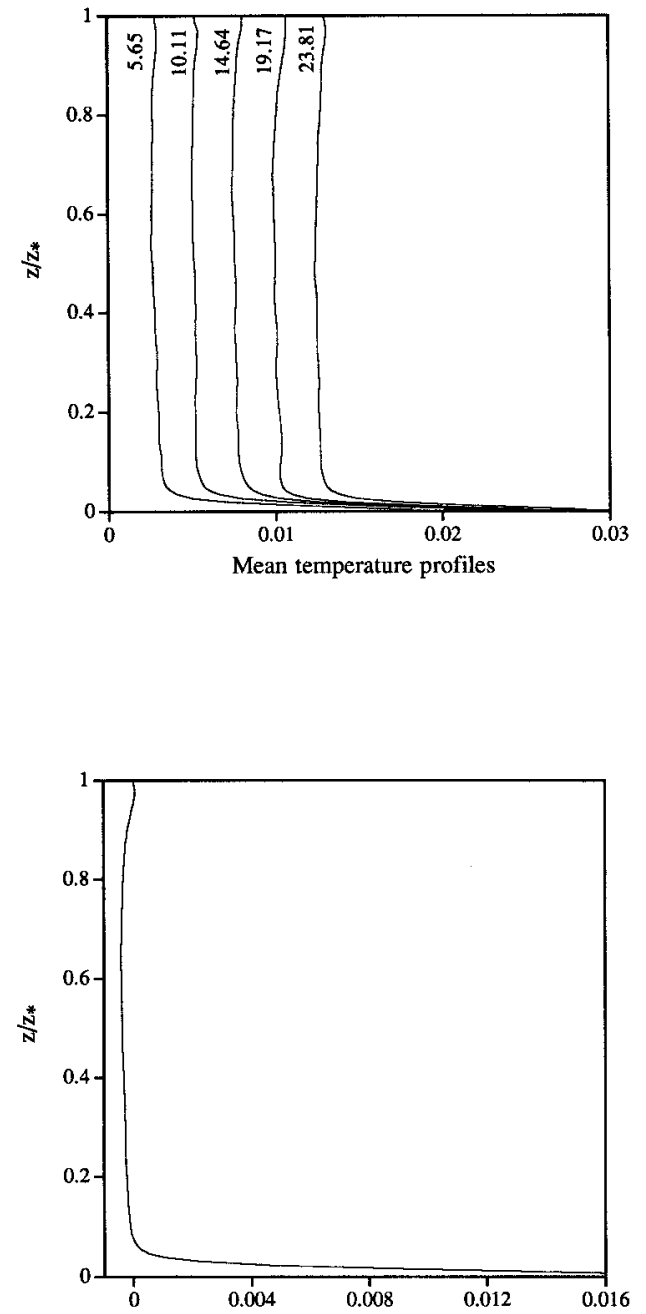

Mean temperature with time rate of change subtracted
Figure 4. Variation of horizontally averaged nondimensional temperature with nondimensional time (time is indicated adjacent to each curve).

Figure 5. Horizontally averaged temperature as a function of $z / z_{*}$ only; the variation in time has been subtracted from each contributing profile.

temperature profiles is displayed in Figure 5. For this particular plot, we have $\theta_{b}=0.01984, \theta_{m}=-0.000306$, and a differential of 0.02 (which, of course, matches the average value of $\left(\theta_{b}-\theta_{m}\right)$ in Figure 3$)$. The average temperature over all $z$ for this profile is -0.000004 , whereas the ideal time rate of change subtracted value should equal 0 . Again, this indicates the high degree of energy conservation in our simulations.

Figure 6 displays two instantaneous temperature contours along horizontal planes, one close to the lower boundary $\left(z / z_{*}=0.0039\right)$, and the other close to the upper one $\left(z / z_{*}=0.9883\right)$. The plane adjacent to the lower boundary lies well inside the conduction layer and is more active; plumes are formed here, and the 


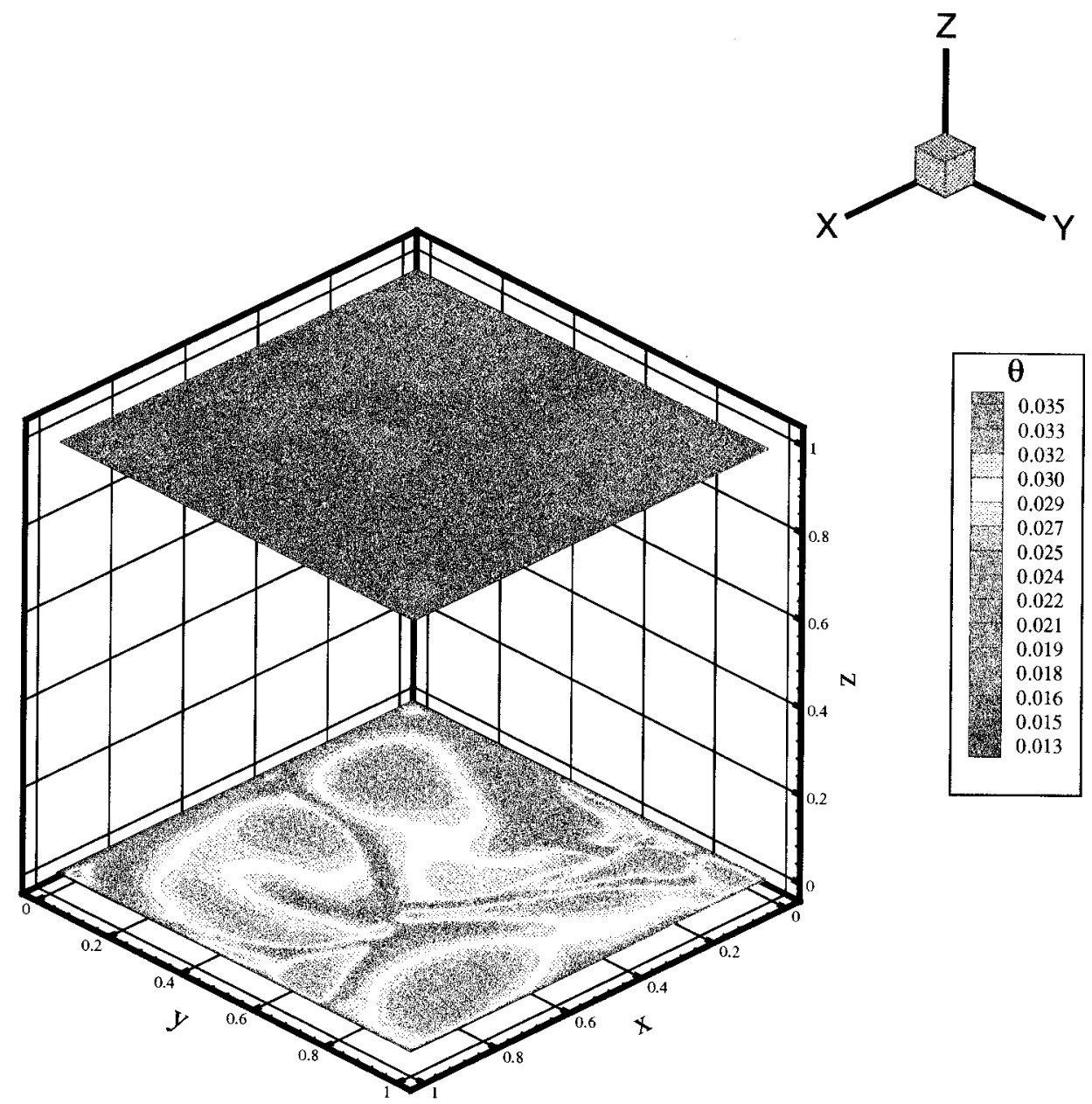

Figure 6. Temperature contours in two horizontal planes: $z / z_{*}=0.0039$ and 0.9883 .

temperature field exhibits a higher degree of fluctuations. Hot ridges of fluid can be seen stretching across the plane closer to the bottom; such ridges are commonly reported in visualization experiments with high Ra convection. The magnitude of the temperature fluctuations is greatly diminished as one approaches the upper boundary. Heat diffuses out of the plumes as they ascend through the enclosure, leaving a rather weak temperature signature in the upper plane.

Figure 7 shows a vertical cut through the enclosure, at plane $y / z_{*}=0.6094$. The temperature contours clearly reveal a mushroom plume; similar plumes are seen at all time steps and are highly reminiscent of the plume visualizations presented by Sparrow et al. $\llbracket 10]$. The plume is clearly seen to originate at the hot ridge forming over the lower boundary. All of these temperature contours closely resemble experimental temperature visualizations with thermochromic liquid crystals performed by the author in nonpenetrative convection. 


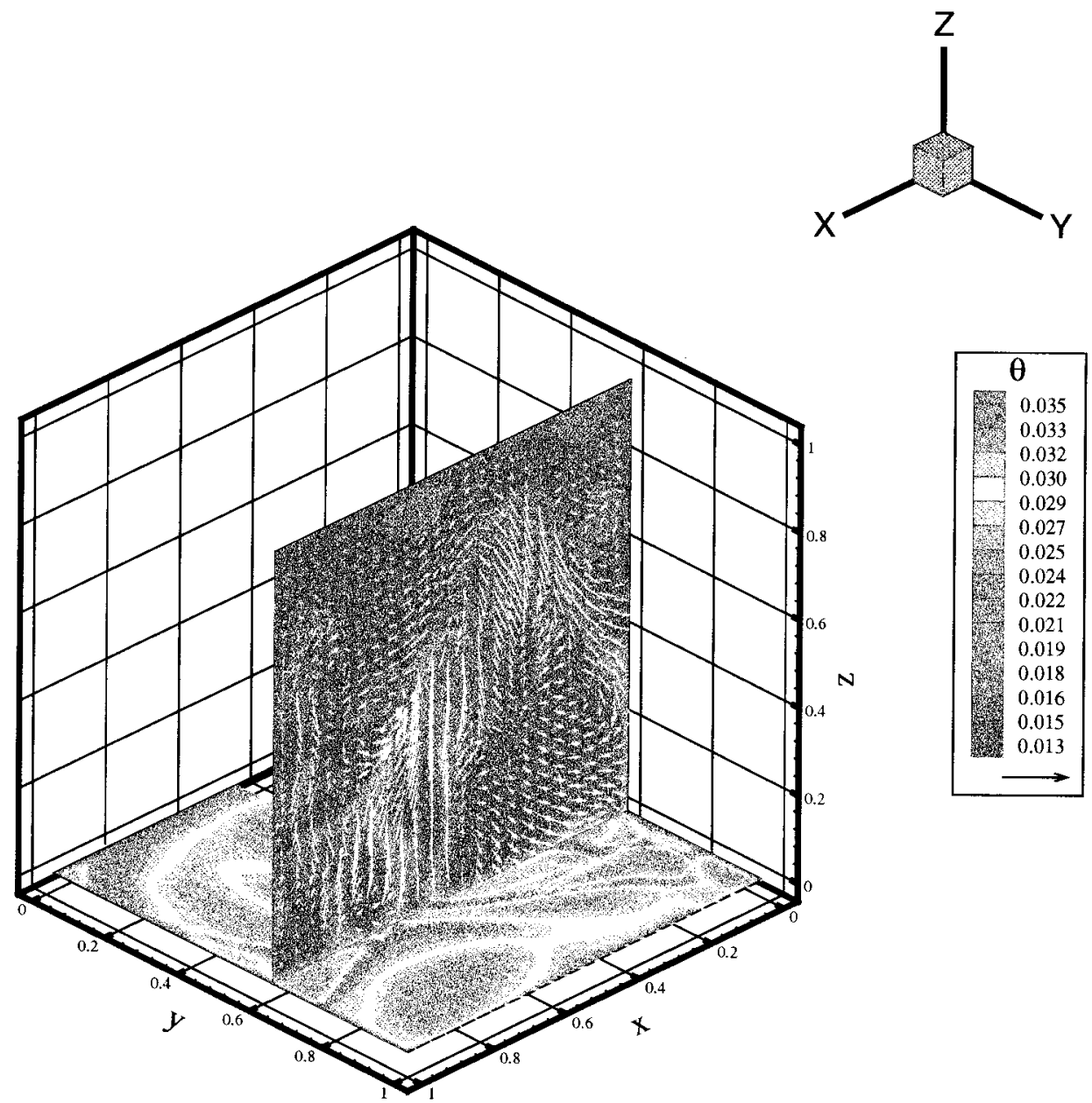

Figure 7. Temperature contours in two planes: $y / z_{*}=0.6094$ and $z / z_{*}=0.0039$; velocity vectors are superimposed (resolution of vectors is $1 / 4$ of simulation resolution).

Velocity vectors are superimposed on the temperature contours in Figure 7. The correlation between the vertical velocity and temperature excursions is obvious. The term "hot plume" is therefore apt. Figure 8 has been included to allow a comparison between simulated and experimental [4] velocity maps. Owing to the highly turbulent nature of the flow, the experimental and simulated vector maps obviously cannot be identical; however, it is useful to note that the size and strength of the flow structures are adequately replicated in the simulation. The resolution of the vector map in Figure 7 is a quarter the resolution employed for the simulation (only every fourth vector is displayed) to prevent saturation of the view field with vectors.

Figure 9 provides a comparison of the simulated root-mean-square (rms) velocity with the experimental measurements of Prasad and Gonuguntla [4]. Note 


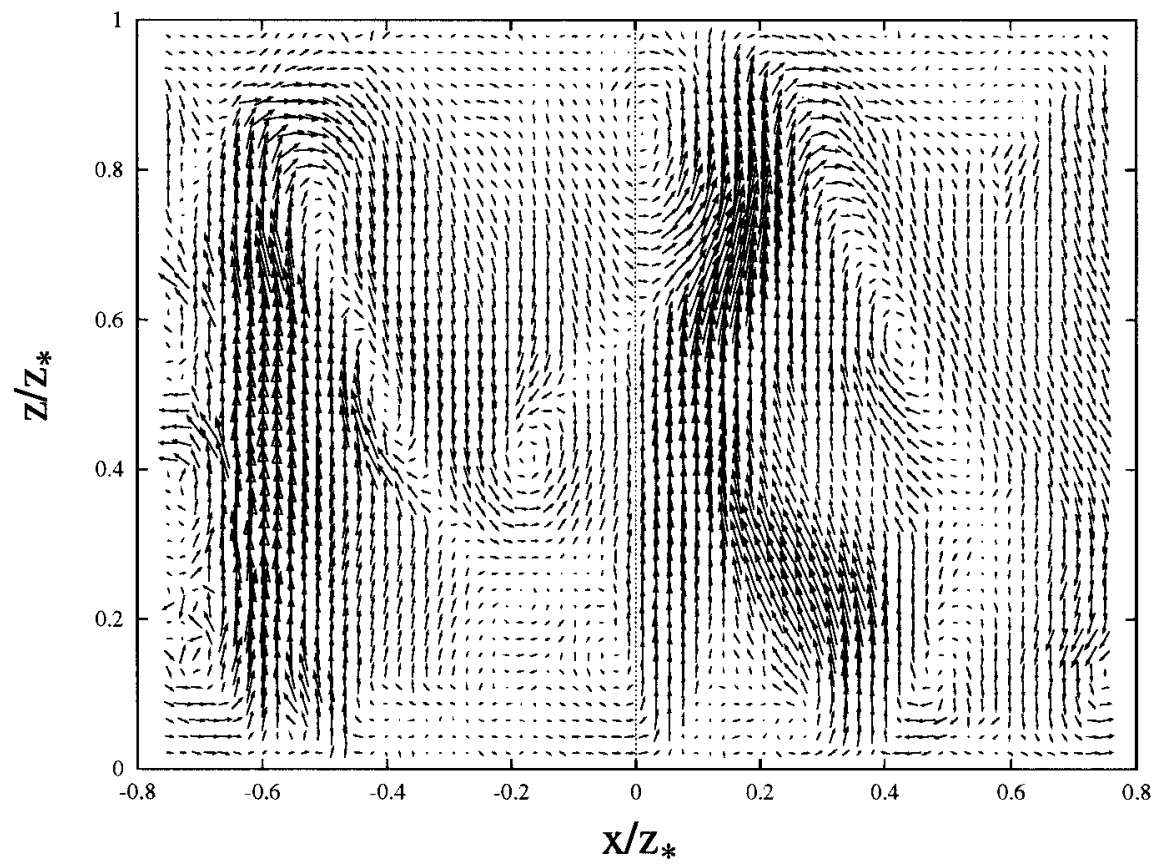

Figure 8. Experimental velocity fields measured by Prasad and Gonuguntla [4] using PIV.

that the mean velocity was not subtracted while computing the rms quantities; the expectation is that the mean velocity should be zero (if the simulation is run for a long enough duration). The horizontal $\left(\sigma_{u}\right.$ and $\left.\sigma_{v}\right)$ velocities are somewhat smaller in the simulation, possibly owing to the disparity in aspect ratio; the simulation was performed with aspect ratio of unity, whereas the experiment used 6.6. Similarly, Adrian et al.'s [6] Laser Doppler Velocimetry (LDV) measurements also yielded horizontal rms velocities in excess of the current simulations; they too used aspect ratios ranging from 7 to 12 . However, the overall shape of the profile with the peaks near the lower and upper boundary is clearly replicated. This peak adjacent to the lower boundary is due to the fact that thermals are produced there and fluid is drawn in horizontally to feed the growth of these structures. The upper peak rises from the impact and horizontal spreading of the rising plumes as they approach the upper boundary.

The $\sigma_{w}$ profiles match quite well. In this case, the peak is located at the midplane owing to the fact that vertical motions are maximized in the region away from the horizontal boundaries. The simulated profile for $\sigma_{\theta}$ is in good agreement with temperature measurements of Adrian et al. [6]. In Figure $9 c, \sigma_{\theta}$ has been normalized by the nondimensional convective temperature scale, $\theta_{*}$, where

$$
\theta_{*}=\left[\mathrm{Ra}^{\prime} \operatorname{Pr}\right]^{-1 / 3}
$$




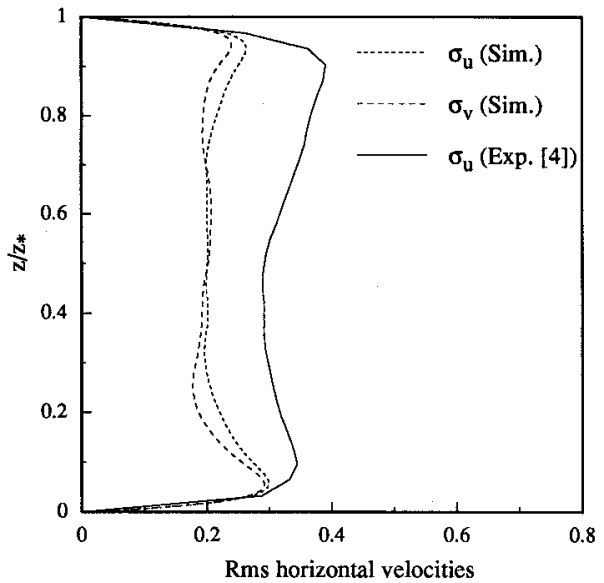

(a)

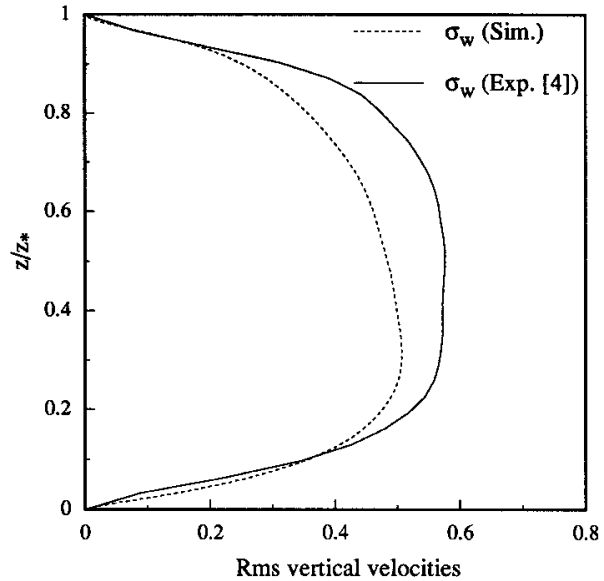

(b)

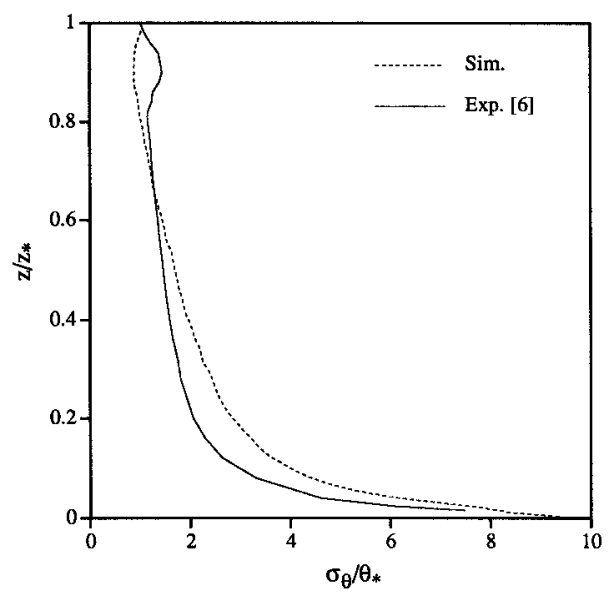

(c)

Figure 9. Comparison between simulated and experimental (a) rms horizontal velocity, $(b)$ rms vertical velocity, and $(c)$ rms temperature.

The above expression for $\theta_{*}$ can be easily derived by applying our nondimensionalization scheme to the dimensional convective temperature scale defined as $T_{*}=$ $q_{0} / \rho C_{p} w_{*}$. For the current simulations, $\theta_{*}=5.414 \times 10^{-4}$.

The simulated skewness of vertical velocity in Figure 10 matches very well with experimental measurements of Prasad and Gonuguntla [4]. The vertical velocity skewness is positive for all $z$, which is expected for nonpenetrative bottom-up convection, which is characterized by less-frequent-but more intense - upward moving hot plumes embedded in cooler, gently descending fluid. Additional details pertaining to the shape of the skewness profile may be found in Prasad and Gonuguntla [4]. 


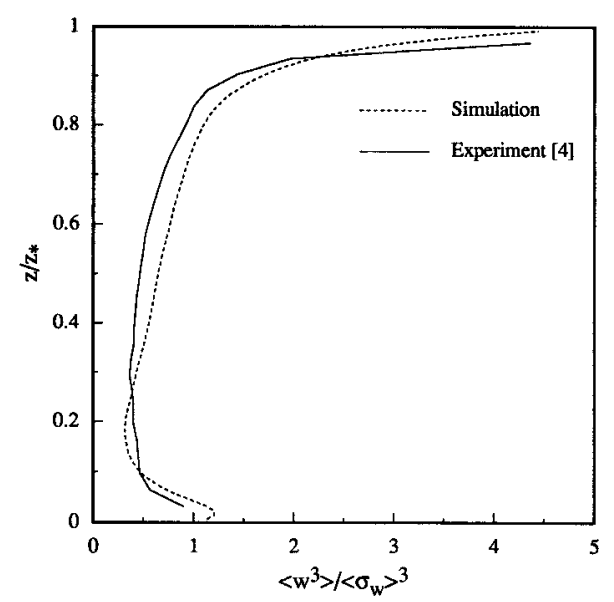

Figure 10. Comparison of simulated velocity skewness with experiment.

\section{Turbulent Heat Flux}

Consider again the nondimensional energy equation Eq. (8). Using Eq. (9), we may write

$$
\frac{\partial \theta}{\partial t}+u_{j} \frac{\partial \theta}{\partial x_{j}}=\left(\frac{1}{\mathrm{Ra}^{\prime} \operatorname{Pr}}\right)^{1 / 3} \frac{\partial^{2} \theta}{\partial x_{j}^{2}}
$$

Using the standard method of Reynolds decomposition, time averaging, and applying horizontal homogeneity, the equation reduces to

$$
\frac{\partial \bar{\theta}}{\partial t}=\left(\frac{1}{\mathrm{Ra}^{\prime} \operatorname{Pr}}\right)^{1 / 3} \frac{\partial^{2} \bar{\theta}}{\partial z^{2}}-\frac{\partial \overline{\theta^{\prime} w}}{\partial z}
$$

But the nondimensional temperature rise is

$$
\frac{\partial \bar{\theta}}{\partial t}=\left(\frac{1}{\mathrm{Ra}^{\prime} \operatorname{Pr}}\right)^{1 / 3}
$$

Using this result, rearranging, and integrating along the vertical coordinate, we obtain

$$
1-z+\frac{\partial \bar{\theta}}{\partial z}=\overline{\theta^{\prime} w}\left(\operatorname{Ra}^{\prime} \operatorname{Pr}\right)^{1 / 3}
$$

The derivative of the temperature profile displayed in Figure 5 was taken with respect to $z$ in order to compute the left-hand side of Eq. (11). Similarly, the profiles for $\overline{\theta^{\prime} w}$ were averaged over all time steps to compute the right-hand side 


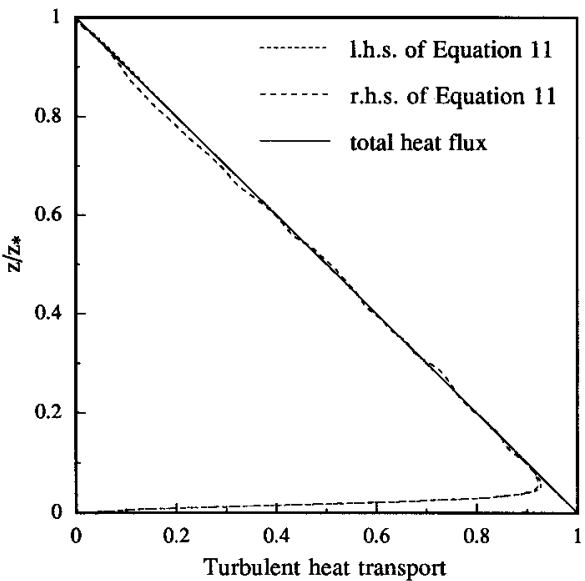

Figure 11. Turbulent heat transport.

of Eq. (11). Figure 11 compares the left- and right-hand sides of Eq. (11). The comparison is excellent, indicating the convergence of the temperature-velocity correlation. The total nondimensional heat flux $q(z) / q_{0}$ is also indicated in the figure as a straight line of slope -1 . The plot reveals that very near the lower boundary, the contribution of turbulence to heat transport is negligible, with molecular diffusion playing the dominant role; obviously, this is the conduction layer. As $z$ increases, the heat transport due to turbulence becomes overwhelmingly large and accounts almost exclusively for the total flux.

\section{CONCLUSIONS}

Nonpenetrative turbulent thermal convection has been investigated at $\mathrm{Ra}$ $2 \times 10^{7}$ in a cubical enclosure using direct numerical simulations with $96 \times 96 \times$ 128 grid points. The numerical results are in good agreement with previous experimental measurements of velocity and temperature. Owing to steady bottom heating, the temperature of the system increases linearly in time. However, it is seen that the horizontally averaged temperature profiles resemble each other to a very high degree. It is the refore reasonable to classify the flow as being quasi-ste ady, in that the unsteadiness is in the form of a linear, predictable increase of temperature over time, while the spatial profile is time invariant. A consequence is that the temperature difference between the bottom and the mixed layer becomes constant after the initial transients have settled down.

The rms velocity profiles are somewhat smaller in the current simulations in comparison to previous experiments. One possible explanation is that these simualtions pertain to a unit aspect ratio, whereas the experiments were carried out at aspect ratios exceeding 6 . The rms temperature and vertical velocity skewness results are in excellent agreement with experimental measurements. Visualizations of temperature and velocity are also in good agreement with experiments. 
The simulations have confirmed Prasad's [5] derivation for the differential between the bottom and mixed layer temperatures in nonpenetrative convection. The derivation requires the convection to be at a high enough Rayleigh number that the upper boundary condition does not significantly influence the lower boundary, i.e., the classical scaling argument that $\mathrm{Nu} \sim \mathrm{Ra}^{1 / 3}$. In the present simulation, $\mathrm{Ra}=2 \times 10^{7}$ and therefore is within the range of applicability of the derivation.

\section{REFERENCES}

1. J. W. Deardorff, G. E. Willis, and D. K. Lilly, Laboratory Investigation of Non-Steady Penetrative Convection, J. Fluid Mech., vol. 35, pp. 7-31, 1969.

2. A. Cenedese and G. Querzoli, A Laboratory Model of Turbulent Convection in the Atmospheric Boundary Layer, Atmos. Environ., vol. 28, pp. 1901-1913, 1994.

3. J. C. Wyngaard and J. C. Weil, Transport Asymmetry in Skewed Turbulence, Phys. Fluids A, vol. 3, pp. 155-162, 1991.

4. A. K. Prasad and P. V. Gonuguntla, Turbulence Measurements in Nonpenetrative Thermal Convection, Phys. Fluids, vol. 8, pp. 2460-2470, 1996.

5. A. K. Prasad, Derivation of Rayleigh Number for Nonpenetrative Thermal Convection, J. Heat Transfer, vol. 119, pp. 180-183, 1997.

6. R. J. Adrian, R. T. D. S. Ferreira, and T. Boberg, Turbulent Thermal Convection in Wide Horizontal Fluid Layers, Exp. Fluids, vol. 4, pp. 121-141, 1986.

7. C.-H. Moeng, and R. Rotunno, Vertical-Velocity Skewness in the Buoyancy-Driven Boundary Layer, J. Atmos. Sci., vol. 47, pp. 1149-1162, 1990.

8. R. M. Kerr, Rayleigh Number Scaling in Numerical Convection, J. Fluid Mech., vol. 310, pp. 139-179, 1996.

9. S. Balachandar, M. R. Maxey, and L. Sirovich, Numerical Simulation of High Rayleigh Number Convection, J. Sci. Comput., vol. 4, pp. 219-236, 1989.

10. E. M. Sparrow, R. B. Husar, and R. J. Goldstein, Observations and Other Characteristics of Thermals, J. Fluid Mech., vol. 41, pp. 793-800, 1970. 